\title{
Serum Exosomal miRNA Might Be a Novel Liquid Biopsy to Identify Leptomeningeal Metastasis in Non-Small Cell Lung Cancer
}

This article was published in the following Dove Press journal: OncoTargets and Therapy

\author{
Qiuli Xu, ${ }^{1, *}$ Liang Ye, ${ }^{2, *}$ \\ Litang Huang,', Li Zhou, ${ }^{3}$ \\ Xi Chen, ${ }^{4}$ Mingxiang Ye, ${ }^{3}$ \\ Guannan Wu, ${ }^{3}$ Ping Zhan, ${ }^{3}$ \\ Tangfeng Lv, ${ }^{3}$ Yong Song \\ 'Department of Respiratory and Critical \\ Care Medicine, Affiliated Jinling Hospital, \\ Southeast University, Sch Med, Nanjing, \\ Jiangsu, People's Republic of China; \\ ${ }^{2}$ Department of Respiratory Medicine, \\ Nanjing First Hospital, Nanjing Medical \\ University, Nanjing, Jiangsu, People's Republic \\ of China; ${ }^{3}$ Department of Respiratory and \\ Critical Care Medicine, Affiliated Jinling \\ Hospital, Medical School of Nanjing \\ University, Nanjing, Jiangsu, People's Republic \\ of China; ${ }^{4}$ Department of Critical Care \\ Medicine, Zhongda Hospital, School of \\ Medicine, Southeast University, Nanjing, \\ Jiangsu, People's Republic of China
}

*These authors contributed equally to this work
Correspondence: Tangfeng Lv

Department of Respiratory and Critical

Care Medicine, Affiliated Jinling Hospital, Medical School of Nanjing University,

\#305, East Zhongshan Road, Nanjing,

People's Republic of China

$\mathrm{Tel} / \mathrm{Fax}+86$ 25-8086-359l

Email bairoushui@I63.com

Yong Song

Department of Respiratory and Critical

Care Medicine, Affiliated Jinling Hospital,

Southeast University, Sch Med, Nanjing,

Jiangsu, People's Republic of China

$\mathrm{Tel} / \mathrm{Fax}+86$ 25-8086-359l

Email yong.song@nju.edu.cn
Purpose: The survival time of patients with leptomeningeal metastasis (LM) of lung cancer is very short, and the clinical characteristics of LM are varied, making the clinical diagnosis difficult. At present, a positive CSF fluid (CSF) cytology result remains the gold standard for diagnosing LM in lung cancer; however, the process of collecting CSF is traumatic and far less convenient than blood collection. With the development in technology, an increasing number of studies prefer to use liquid biopsy to diagnose or predict the occurrence of the disease. Therefore, we aimed to explore whether serum exosomal miRNA can replace miRNA from CSF to identify or predict the occurrence of LM.

Patients and Methods: Herein, four pairs of serum and CSF samples were collected at four different time points from a patient with LM from non-small cell lung cancer (NSCLC). Serum and CSF exosomes were extracted. Western blot (CD63, TSG101) and electron microscope analyses were used to verify exosome extraction, after which exosomal miRNA sequencing was performed. Next, exosomal miRNA from serum and CSF samples from seven patients with LM and 30 patients without LM were collected for validation.

Results: Sequencing results of serum exosomal miRNA and CSF exosomal miRNA showed that there were 44 exosomal miRNAs stably co-expressed at four different time points. Then, three common miRNAs related to LM were found (hsa-miR-483-5p, hsa-miR-423-5p, and hsa-miR-342-5p). Subsequently, exosomal miRNA was extracted from serum and CSF samples from seven patients with LM and 30 patients without LM for verification, and the expression of these exosomal miRNA was detected. The results showed that miRNA-483-5p and miRNA-342-5p significantly differed in LM- $/+$ patients $(\mathrm{P}=0.0142$ and $\mathrm{P}=0.0031$, respectively), whereas miRNA-423-5p had no difference $(\mathrm{P}=0.0921)$. Additionally, as the symptoms improved, the expression of these miRNAs decreased or remained stable.

Conclusion: Serum exosomal miRNA (hsa-miR-483-5p, and hsa-miR-342-5p) may be involved in LM of lung cancer and may replace CSF to predict LM in NSCLC.

Keywords: non-small cell lung cancer, leptomeningeal metastasis, exosome, miRNA, liquid biopsy

\section{Introduction}

The incidence and mortality of lung cancer in China ranks first among malignant tumors and leptomeningeal metastasis is one of the rare but most serious complications of nonsmall cell lung cancer (NSCLC). ${ }^{1}$ The incidence of leptomeningeal metastasis of NSCLC ranges from $3 \%$ to $5 \% .^{2}$ Once leptomeningeal metastasis occurs, the disease progresses rapidly and the prognosis is extremely poor, which greatly shortens the survival time of lung cancer patients. 
Leptomeningeal metastases vary in clinical symptoms, including symptoms of high intracranial pressure such as headache, nausea, and vomiting, and symptoms of brain parenchyma and neurological involvement, such as limb movement disorders, hearing loss, psychiatric symptoms, and seizures. ${ }^{3,4}$ The varied clinical presentation can make the diagnosis more difficult. At present, the diagnosis of leptomeningeal metastasis is mainly based on the clinical manifestations of neurological damage, imaging, and CSF cytology. CSF cytology is still the gold standard for the diagnosis of leptomeningeal metastases. ${ }^{4}$ The sensitivity of the first lumbar puncture is as low as $50 \%$, and it can be increased to $75-85 \%$ by the second CSF analysis. ${ }^{5}$ To improve sensitivity, repeated collection of CSF samples through a lumbar puncture is performed, taking at least $10 \mathrm{~mL}$ of CSF at a time and quickly analyzing it. $^{2}$ Leptomeningeal biopsy is rarely used to confirm the diagnosis as this invasive procedure can cause extensive damage to the patient. With the development in technology, more and more researchers tend to use liquid biopsy to diagnose or predict the occurrence of the disease, so as to curb or delay the adverse outcome in the very early stage of the disease. ${ }^{6}$

Exosomes are 50-200 $\mathrm{nm}$ extracellular vesicles (EVs) that are secreted by cells. ${ }^{7}$ Several studies have shown that microRNAs (miRNAs) plays crucial roles in various cancer-related pathological processes by acting as a messenger between lung cancer cells and other cells, which undoubtedly represents a breakthrough in the discovery of new biomarkers and therapeutic targets in lung cancer. ${ }^{33}$ Rodriguez et al characterized the expression of exosomal miRNAs in the plasma of NSCLC and nontumor patients and found that the expression of exosomal miR-141 in NSCLC patients was significantly lower than that in non-tumor patients. ${ }^{34}$ Zhang et al found that the expression level of serum exosomal miR-17-5p in NSCLC patients was higher than that in healthy people, suggesting that serum exosomal miR-17-5p can be used as a diagnostic biomarker for NSCLC. ${ }^{35}$ It has been reported that exosomal miRNAs can also be used as biomarkers to distinguish different types of NSCLC. In another study, four exosomal miRNAs (miR181-5p, miR-30a-3p, miR$30 \mathrm{e}-3 \mathrm{p}$, and miR-361-5p) and three exosomal miRNAs (miR-10b-5p, miR-15b-5p and miR-320b) specific to adenocarcinoma and squamous cell carcinoma, respectively, were identified. ${ }^{36}$ Recent research suggests that exosomal miRNAs are related to a variety of pathological processes in lung cancer, such as cell proliferation and migration, angiogenesis, and epithelial-mesenchymal transition (EMT). ${ }^{28}$ Accumulating evidence indicates that miRNAs may be involved in the processes of invasion and metastasis in lung cancer. ${ }^{37}$ Additionally, exosomal miR-193a$3 p$, miR-210-3p, and miR-5100 secreted by hypoxic bone marrow-derived mesenchymal stem cells could enhance the invasion and metastasis of lung cancer cells through activation of STAT3 signaling, inducing EMT in these cancer cells. ${ }^{38}$ Some EVs located in body fluids are rich in miRNAs that are involved in brain metastases. In 2017, Fan et al discovered that exosomal miRNAs in the CSF of NSCLC patients with LM were involved in the progression of LM and could be used as potential diagnostic tools. ${ }^{8}$ CSF specimen collection is challenging as it can cause more trauma and inconvenience to the patient than blood collection. As such, we aimed to identify serum exosome-specific miRNA profiles in NSCLC patients with LM that can be used to predict or identify the occurrence of LM, thus, replacing CSF miRNA profiling as a diagnostic technique.

\section{Patients and Methods Sample Collection}

This study was approved by the Ethics Committee of Jinling Hospital. Written and informed consent was obtained from all patients and healthy volunteers, and this study was conducted in accordance with the Declaration of Helsinki. Serum and CSF specimens from eight patients with leptomeningeal metastases from lung cancer were collected from August 2018 to January 2020 at Jinling Hospital, Nanjing University. We selected one patient with leptomeningeal metastasis (LM) for longitudinal tracing of changes in serum exosomes; the other seven patients with leptomeningeal metastases and 30 without leptomeningeal metastases were assigned to the validation cohort.

\section{Extraction of Exosomes from the Serum and CSF}

Overall, $4 \mathrm{~mL}$ of blood samples were collected into plain tubes, allowed to clot at $37^{\circ} \mathrm{C}$ for $20 \mathrm{~min}$, and centrifuged at $2000 \times \mathrm{g}$ for $10 \mathrm{~min}$ to collect the serum. The clear serum was further centrifuged at $10,000 \times \mathrm{g}$ for $30 \mathrm{~min}$ to remove cells and debris. The exosomes were isolated according to the manufacturer's protocol. Following this, $400 \mu \mathrm{L}$ of the recently centrifuged serum was transferred to a new tube and 0.2 volumes of the Total Exosome 
Isolation reagent were added. The serum/reagent mixture was mixed well by eddying until there was a homogenous solution. The sample was then incubated at $4{ }^{\circ} \mathrm{C}$ for 30 min. After incubation, the samples were centrifuged at $10,000 \times \mathrm{g}$ for $10 \mathrm{~min}$ at room temperature. The supernatant was discarded and the pellet, which contained the exosomes, at the bottom of the tube was resuspended in $200 \mu \mathrm{L}$ phosphate-buffered saline (PBS). CSF exosomes were extracted using the classic exosome separation method.

\section{Transmission Electron Microscopy (TEM)}

The isolated exosomes were resuspended in PBS. The suspension was placed on a chloroform-coated copper grid with $0.125 \%$ Formvar and negatively stained with uranyl acetate. Images were observed under a JEOL 1200EX TEMSCAN electron microscope (Japan).

\section{Western Blotting}

The procedures were conducted as previously described. ${ }^{9}$ The isolated exosome pellet was lysed using a lysis buffer containing the protein extraction reagent RIPA (Beyotime, Shanghai, China), PMSF (Roche, Basel, Switzerland), and a protease inhibitor cocktail (Roche, Basel, Switzerland). The BCA protein assay kit (Thermo Scientific, Rockford, Illinois, USA) was used to quantify the total protein concentration. Approximately $30 \mu \mathrm{g}$ of total protein was electrophoresed on a $10 \%$ sodium dodecyl sulfatepolyacrylamide gel and electro-transferred to a PVDF membrane (Millipore, Shanghai, China). The membrane was then blocked with $5 \%$ skimmed milk for $2 \mathrm{~h}$, immunoblotted with anti-CD63 (1:1000; Abcam, Cambridge, UK), anti-TSG101 (1:1000; Abcam, Cambridge, UK), and GAPDH primary antibody (1:2000; Abcam, Cambridge, UK), and incubated with the secondary antibody for $60 \mathrm{~min}$.

\section{Cell Lines, Cell Culture and Transfection}

The human LUAD cell line H1975 was purchased from Shanghai Institute of Life Sciences (Shanghai, China). The cells were cultured in RPMI 1640 medium (KeyGene, Nanjing, China), containing $10 \%$ fetal bovine serum (FBS) and penicillin/streptomycin (KeyGene, Nanjing, China) in a humidified environment with 5\% CO2 at 37 ${ }^{\circ} \mathrm{C}$. Cells were seeded in 6-well plates before transfection. When the fusion rate reaches $60 \%-70 \%$, use Lipofectamine 3000 reagent (Invitrogen, California, USA) to transfect MiRNA mimic (10nM, 50nM) and negative control into the cells. The miRNA mimics and mimic negative control were purchased from RiboBio (Guangzhou, China). Transfection efficiency was assessed by quantitative real-time RT-PCR.

\section{RNA Isolation and qRT-PCR}

Total RNA was extracted from exosome with TRIzol reagent (Invitrogen, California, USA). The measurements were assessed with $\triangle \mathrm{CT}$ which was normalized by U6 snRNA (RiboBio, Guangzhou, China). The expression levels of miR-483-5p, miR-423-5p, and miR-342-5p were measured via qRT-PCR using a Bulge-Loop ${ }^{\mathrm{TM}}$ miRNA reverse transcription kit (RiboBio, Guangzhou, China), and a Bulge-Loop ${ }^{\mathrm{TM}}$ miRNA qRT-PCR starter kit (RiboBio, Guangzhou, China). U6 snRNA (RiboBio, Guangzhou, China) was used as an endogenous control to quantify and normalize the results. The value $2^{-\Delta \Delta C T}$ was used for comparative quantitation. All qRT-PCR reactions were performed in triplicate.

\section{Exosome RNA-Sequencing}

Exosome total RNA was reverse transcribed to cDNA and amplified using Discover-scTM WTA kit V2 (Vazyme, Nanjing, China) according to the manufacturer's instructions. The amplified cDNA was quantified using QuBit (Life Technologies Pvt Ltd., Delhi, India) and quality control was performed using Agilent 2100 Bioanalyzer (USA) with High Sensitivity DNA Chip. Sample preparation was done via Biorupter shearing through sonication. Sequencing was performed using the TruePrep TM DNA Library Prep Kit V2 for Illumina ${ }^{\circledR}$ (Vazyme, Nanjing, China). Size-selection of $350 \mathrm{bp}$ was done on the Illumina $\mathrm{X}$ ten platform.

\section{Nanoparticle Tracking Analysis (NTA)}

Isolated exosomes were uniformly diluted in PBS solution and were further measured using a NanoSight NS300 Instrument (NanoSight Ltd, Amesbury, UK) with NTA software. Approximately $3 \times 10^{8}$ particles $/ \mathrm{mL}$ of sample was used to assess the size distribution and concentration.

\section{Statistical Analyses}

All experiments were conducted in triplicate, and the data were analyzed using SPSS 23.0 software. Student's $t$-test and ANOVA test were applied to determine statistically significant differences $(\mathrm{P}<0.05)$ between different groups. 

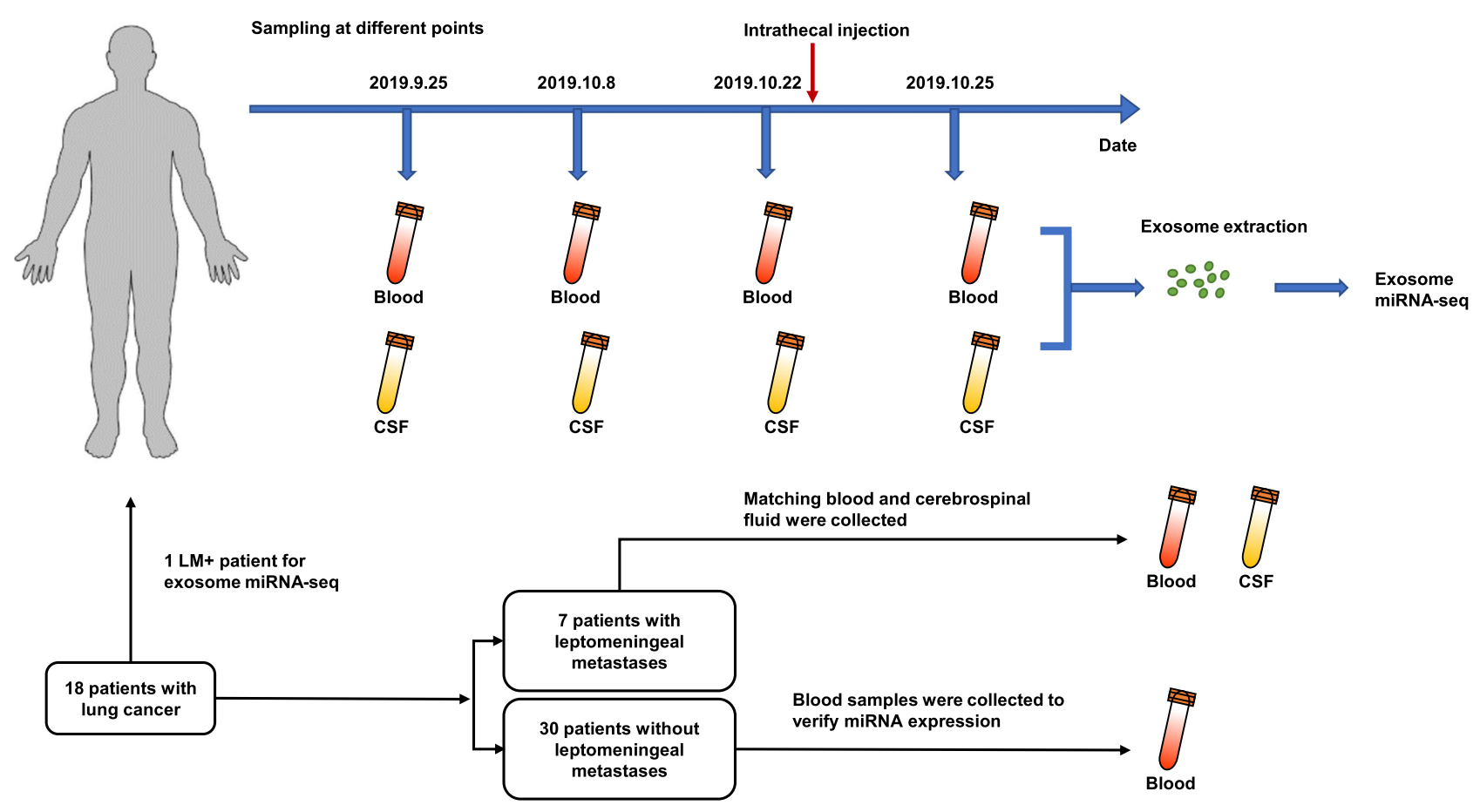

Figure I Flowchart depicting the experimental design and sample collection.

\section{Results}

\section{Samples of Study Cohort}

We selected a patient with leptomeningeal metastases from NSCLC. At four different time points during the patient's visit, the patient's serum and CSF were simultaneously obtained. The exosomes in the serum and CSF were extracted for examination, and RNA in the exosome was extracted for further sequencing analysis (Figure 1). We enrolled additional seven patients with leptomeningeal metastases and assigned them to the validation cohort. We also included 30 patients without leptomeningeal metastases; their characteristics are summarized in Tables 1 and 2.

\section{Characterization of Exosomes Extracted from Serum}

As there was a limited volume of serum from each collection, we used the precipitation method to extract the exosomes from the serum. However, the CSF has a relatively large sample volume and relatively few exosomes, which prompted us to send the samples to a professional company for extraction. Next, we used the size, morphology, and markers of the exosomes to detect the isolated exosomes.

Exosomes are spherical, membrane-bound vesicles (Figure 2A). ${ }^{10}$ NTA showed that the diameter of the exosomes extracted was $190.7 \mathrm{~nm}$ (Figure 2B) and Western blotting was able to generate a rich expression of exosomal-specific markers, namely CD63 and TSG101 (Figure 2C). In summary, these methods proved that the exosomes were successfully extracted.

\section{MiRNA Expression Profiles of CSF Exosomes and Serum Exosomes at Different Stages}

The sequencing results from the four time points of serum and CSF collection show that for the same patient, the overall expression level of miRNA in serum exosomes is higher than that in CSF (Figure 3A). Therefore, it is easier to predict the occurrence of leptomeningeal metastasis with miRNA in serum exosomes instead of CSF exosomes.

Studies have shown that LM patients have 181 specific miRNAs (129 upregulated and 52 downregulated) compared with non-meningioma patients with metastases and normal people. ${ }^{11}$ Therefore, the application of miRNA in patients with LM has value in research. We found 44 miRNAs are co-expressed and stable in blood and CSF exosomes using a Venn diagram (Figure 3B). Through an extensive literature retrieval, we found that three of these intersection miRNAs were related to leptomeningeal metastasis, namely miRNA483-5p, miRNA -423-5p, and miRNA -342-5p, ${ }^{8,11}$ which led us to study these three miRNAs. 
Table I Baseline Demographic and Clinical Characteristics of LM+ Patients

\begin{tabular}{|l|l|l|}
\hline Characteristics & Total(N=8) & $\%$ \\
\hline Age(years), median range & 59.8 & $(41-72)$ \\
$<65$ & 4 & 50 \\
$\geq 65$ & 4 & 50 \\
\hline Gender & & \\
Male & 6 & 75 \\
Female & 2 & 25 \\
\hline Smoking status & & \\
Former/current & 4 & 50 \\
Never & 4 & 50 \\
\hline Histology & & \\
Adenocarcinoma & 6 & 75 \\
Other & 2 & 25 \\
\hline EGFR mutation & & \\
Yes & 6 & 75 \\
No & 0 & 0 \\
Unknown & 2 & 25 \\
\hline Metastasis site & & 100 \\
Leptomeningeal (yes/no) & 8 & 50 \\
Brain(yes/no) & 4 & 25 \\
Lung(yes/no) & 5 & \\
Bone(yes/no) & 3 & \\
Liver(yes/no) & & \\
Pericardium(yes/no) & & \\
Pleura(yes/no) & & \\
\hline
\end{tabular}

Abbreviation: LM, leptomeningeal metastasis.

\section{Differences Between Patients with or without Leptomeningeal Metastasis of the Target miRNA}

Based on previous sequencing and literature searches, we focused on the three miRNAs, miRNA-483-5p, miRNA423-5p and miRNA-342-5p. We recruited seven patients with leptomeningeal metastasis and 30 patients without leptomeningeal metastasis to verify the expression of these three miRNAs. The results show that miRNA-483$5 p$ and miRNA-342-5p have significant differences in leptomeningeal and non-leptomeningeal metastasis patients $(\mathrm{P}=0.0142$ and $\mathrm{P}=0.0031$, respectively; Figure $4 \mathrm{~A}$ and $\mathrm{C}$ ), whereas miRNA-423-5p shows no difference $(\mathrm{P}=0.0921$, Figure 4B). The expression of miRNA-483$5 p$ and miRNA-342-5p in serum exosomes of patients with leptomeningeal metastasis was higher than the expression seen in non-leptomeningeal metastasis patients. Interestingly, we also found that with disease progression,
Table 2 Baseline Demographic and Clinical Characteristics of LM- Patients

\begin{tabular}{|l|l|l|}
\hline Characteristics & Total(N=30) & $\%$ \\
\hline Age(years), median range & 61 & $(35-8 \mathrm{I})$ \\
$<65$ & 17 & 57 \\
$\geq 65$ & 13 & 43 \\
\hline Gender & & \\
Male & 14 & 47 \\
Female & 16 & 53 \\
\hline Smoking status & & \\
Former/current & 11 & 37 \\
Never & 19 & 63 \\
\hline Histology & & \\
Adenocarcinoma & 19 & 63 \\
Other & 11 & 37 \\
\hline EGFR mutation & & \\
Yes & 6 & 20 \\
No & 7 & 23 \\
Unknown & 17 & 57 \\
\hline Metastasis site & & 17 \\
Leptomeningeal (yes/no) & 0 & 0 \\
Brain(yes/no) & 5 & 20 \\
Lung(yes/no) & 5 & \\
Bone(yes/no) & 0 & \\
Liver(yes/no) & 6 & \\
Pericardium(yes/no) & & \\
Pleura(yes/no) & & \\
\hline
\end{tabular}

Abbreviation: LM, leptomeningeal metastasis.

the expression of the three miRNAs in serum gradually increased. When the disease is no longer metastatic, the expression of miRNAs decreases or remains stable (Figure 4D-F). However, considering the limitations of the small sample size in this study, we recommend obtaining more samples for further verification in the future.

\section{Discussion}

Exosomes are extracellular vesicles found in blood, urine, and other body fluids. ${ }^{12,13}$ They can be secreted excessively by tumor cells and healthy cells. One obvious difference is that exosomes from cancer cells contain more miRNAs than healthy cells, and the exosomes from cancer cells are unique from those in healthy cells. ${ }^{27}$ As key information transmitters, exosomes are rich in biologically active molecules. Their rich nucleic acid and protein content make them potentially valuable biomarkers for non-invasive molecular diagnostics. ${ }^{16,25}$ Sometimes, they are secreted by tumor 
A

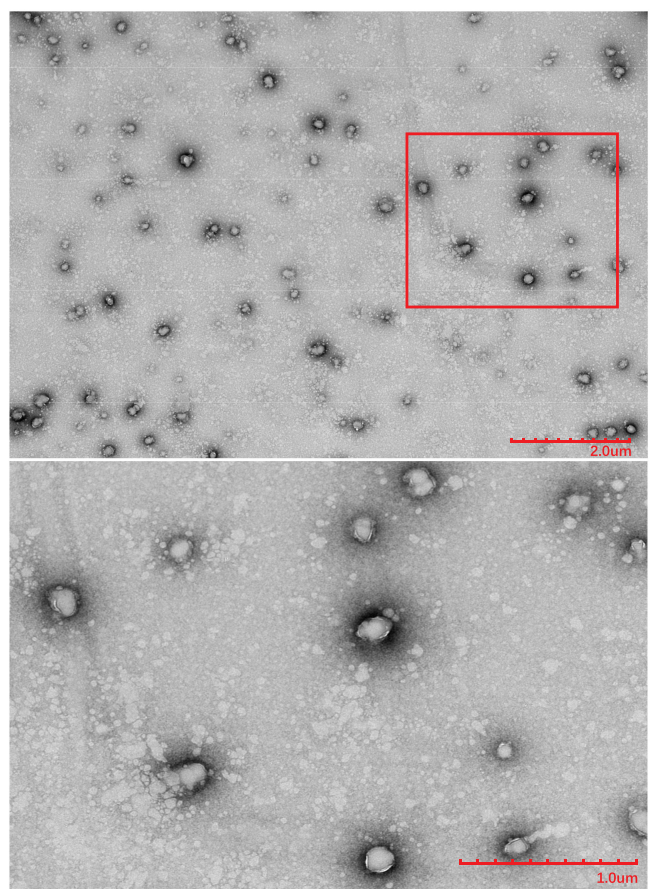

B

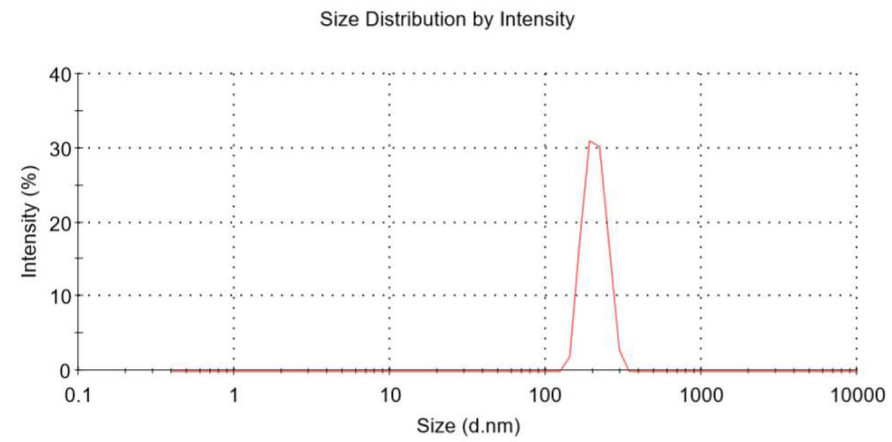

C

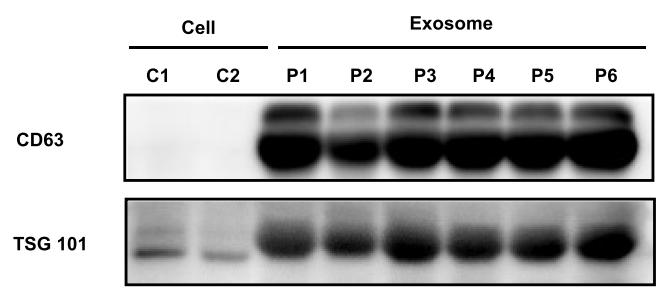

Figure 2 Characterization of exosomes. (A) Morphological characterization of exosomes isolated from serum samples by transmission electron microscopy(TEM). Typical exosomes were amplified. Scale bar $=2 \mu \mathrm{m}$ (upper), I $\mu \mathrm{m}$ (lower). (B) Nanoparticle tracking analysis (NTA) analysis revealed that the average size distribution of exosomes was $190.7 \mathrm{~nm}$. (C) Western blot analysis of exosome protein markers (CD63 and TSGI0I).

A

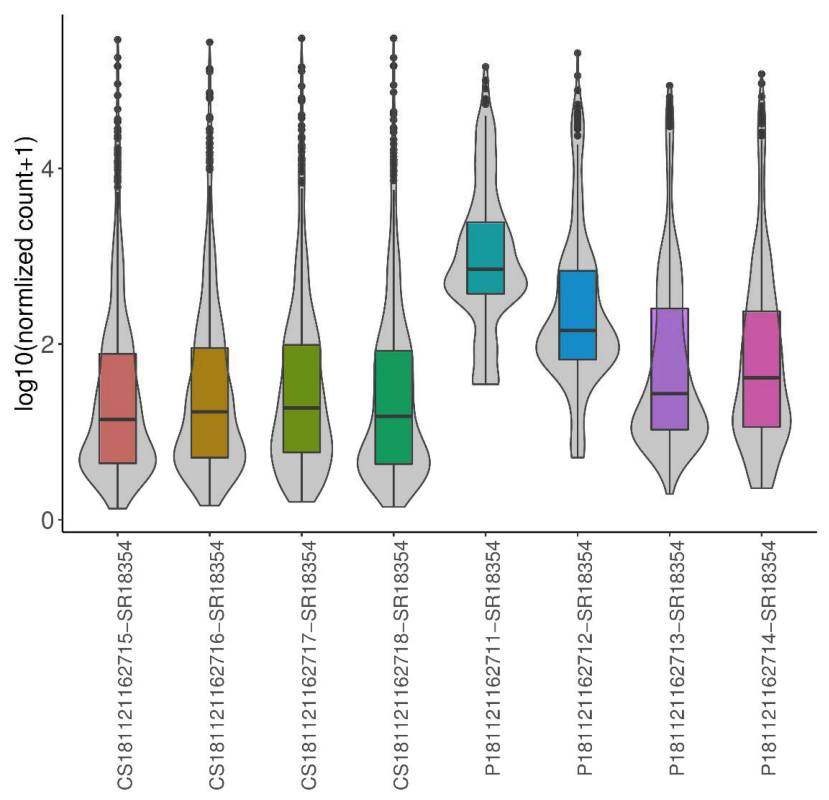

B

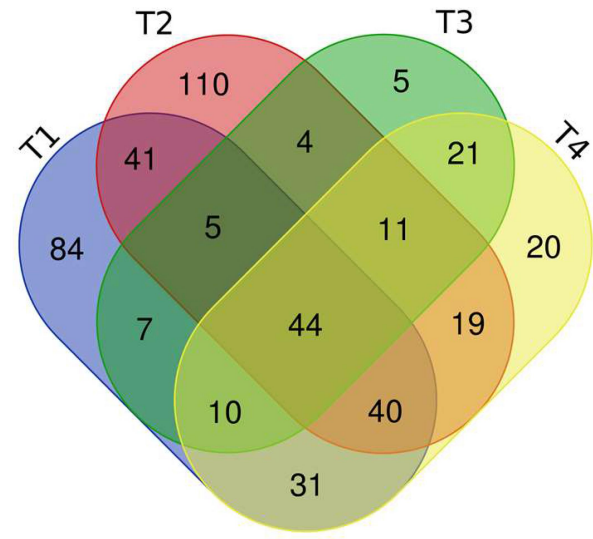

Figure 3 Exosomal miRNA analysis. (A) MiRNA expression levels in the sequenced samples. (B) MiRNAs were co-expressed in CSF and serum exosomes at 4 time points. $\mathrm{T}=$ time.

cells under oxidative stress and participate in cross-talk with the cancer microenvironment. ${ }^{14,15}$ In fact, circulating exosomes have been known to have certain clinical potential and have been able to regulate biological functions in tumors, including immune checkpoint molecules. ${ }^{19-21}$ It has been confirmed that cancer cells release exosomes that carry multiple genetic information. ${ }^{17-21,27}$ More and more studies have shown that tumor-derived miRNAs are closely related to the 
A

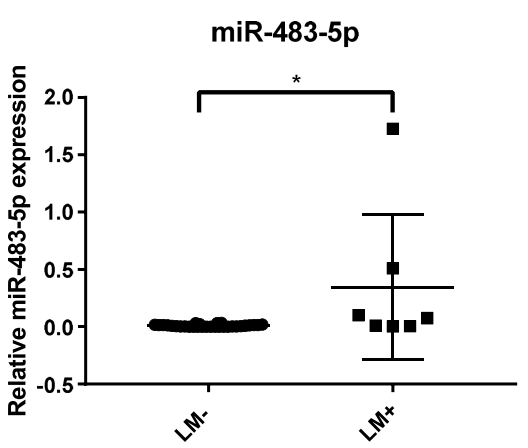

D

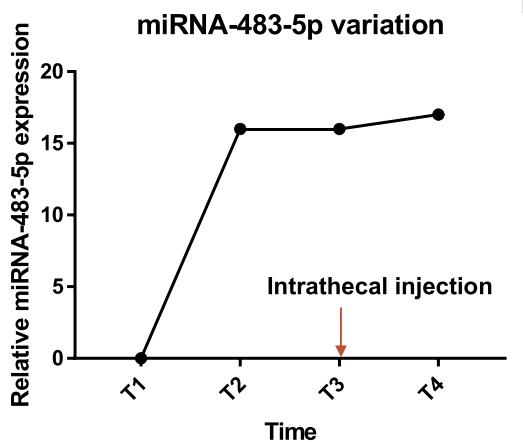

B

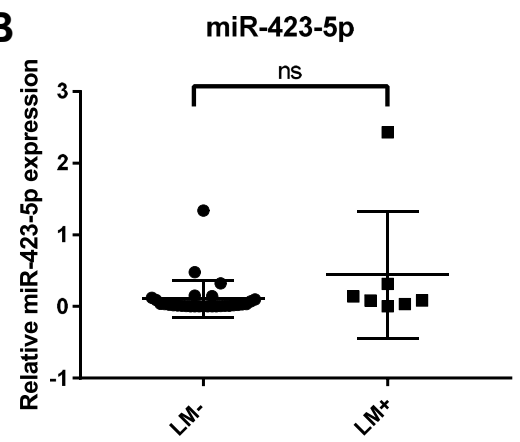

E

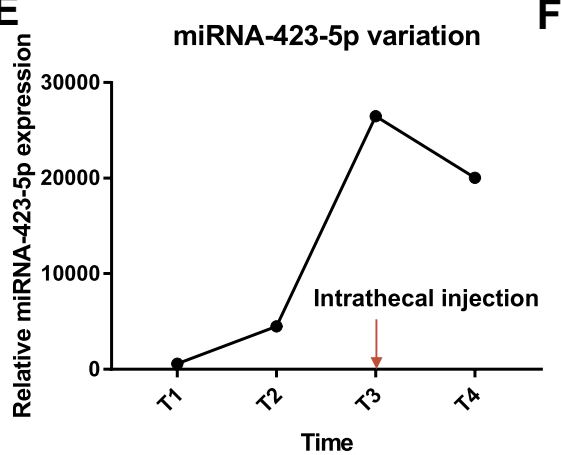

C

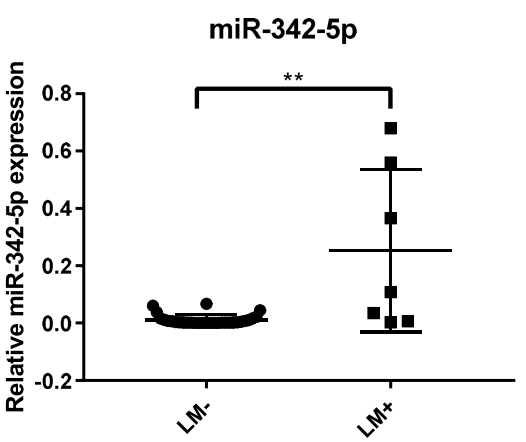

$\mathbf{F}$

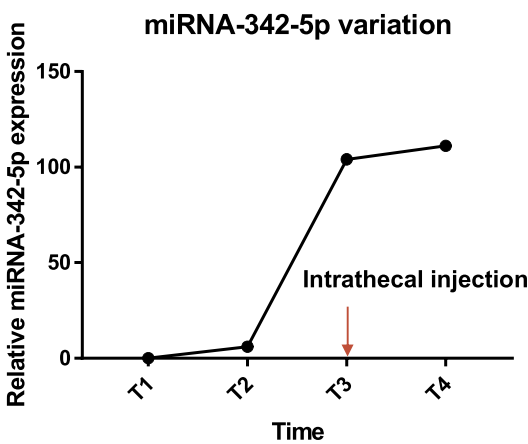

Figure 4 Assessment of miRNA expression levels using quantitative reverse-transcription PCR (qRT-PCR). Relative expression levels of miRNA-483-5p (A), miRNA-423-5p (B), and miRNA-342-5p (C), in LM-1+ patients. U6 snRNA served as the internal control. $* \mathrm{P}<0.05$. $* * \mathrm{P}<0.01$. Changes in miRNA-483-5p (D), miRNA-423-5p (E), and miRNA-342-5p (F) expression over time. Intrathecal injection: methotrexate $10 \mathrm{mg}+$ dexamethasone $5 \mathrm{mg}$; ns = non-significant.

immunosuppressive tumor microenvironment, effector cell abnormalities, and tumor immunogenicity decline. ${ }^{22,26}$

Our data are consistent with previous studies, which confirms that miRNA-483-5p is present in CSF circulating exosomes of LM patients. ${ }^{11}$ In addition, miRNA-483-5p was also found in the serum exosomes. Besides, we found that serum exo-miRNA-483-5p levels of LM patients were higher than those in the control group.

Using three miRNA target prediction programs (TargetScan: targetscan.org, DIANA TOOLS: diana.imis. athena-innovation.gr/DianaTools/index.php,

and miRWALK: mirwalk.umm.uni-heidelberg.de), miRNA483-5p was predicted to target the $A L C A M$ gene. We overexpressed miRNA-483-5p by using miRNA mimic (Figure S1A). We further proved that miRNA-483-5p overexpression inhibits ALCAM expression (Figure S1B), which is consistent with the article written by Song et al (2014). ${ }^{23}$ ALCAM has been confirmed to be crucial to the bloodbrain barrier (BBB) according to Lécuyer et al (2017), and it was confirmed that the BBB of ALCAM KO mice had a higher permeability coefficient, which indicated that tumor cells may easily penetrate the BBB and enter the central nervous system. ${ }^{24}$ We hypothesized that exosomes released by NSCLC patients contained more miRNA-483$5 \mathrm{p}$, which inhibited the expression of ALCAM when transported to the vicinity of $\mathrm{BBB}$, thereby reducing the permeability of the $\mathrm{BBB}$ and making it easier for circulating cancer cells to spread to the central nervous system via the BBB.

We also found that the expression of miRNA-483-5p and miRNA-342-5p was significantly higher in the serum exosomes of patients with LM than in the control group. miRNA-342-5p can act as a downstream effector of Notch signaling, regulating the differentiation of neural stem cells (NSCs) to intermediate neural progenitors and astrocytes. $^{29}$ The BBB is formed by capillary endothelial cells surrounded by basal lamina and astrocytic perivascular endfeet. The specialized foot-processes of perivascular astrocytes are in close proximity to the outer surface of brain microvessels and have specialized functions in inducing and regulating the BBB permeability. ${ }^{30}$ Astrocytes promote ions, amino acids, neurotransmitters, and hydrohomeostasis in a variety of ways, and perivascular endfoot astrocytes have a specific role in these processes during BBB formation. ${ }^{39}$ Strong evidence from cell culture studies suggests that astrocytes can upregulate many 
BBB features, leading to tighter tight junctions (physical barriers). ${ }^{31,32} \mathrm{miR}-342-5 \mathrm{p}$ may inhibit the differentiation of NSCs/intermediate progenitor cells into astrocytes through direct targeting of glial fibrillary acidic protein. The overexpression of miR-342-5p results in a significant decrease in the number of astrocytes ${ }^{29}$ and, thus, may affect BBB permeability and LM.

In addition, compared with CSF, serum was easier to obtain and caused less trauma to patients, which were two factors considered when monitoring changes in the disease. The miRNAs from exosomes have become tools for the early identification and prevention of LM, and may increase the survival of NSCLC patients with leptomeningeal metastasis. In this study, we found that miRNA-483$5 p$ and miRNA-342-5p coexist stably in blood and CSF, and may recognize leptomeningeal metastasis and participate in the process of lung cancer leptomeningeal metastasis. Due to the scarcity of leptomeningeal metastasis samples, we have not been able to obtain more samples for verification. There are still many questions to explore, such as, where are these miRNAs released? Is it released from metastasis into the blood, or released from the primary site into the CSF through the $\mathrm{BBB}$, and what cells, tumor cells, or immune cells were involved? In the future, more studies are needed to explore the specific roles and mechanisms of these miRNAs in leptomeningeal metastasis.

\section{Conclusion}

Serum exosomal miRNA (hsa-miR-483-5p, and hsa-miR $-342-5 p$ ) may be involved in LM of lung cancer and may replace CSF to predict LM in NSCLC.

\section{Acknowledgments}

We are grateful to all the participants who made this research possible.

\section{Author Contributions}

All authors made a significant contribution to the work reported, whether that is in the conception, study design, execution, acquisition of data, analysis and interpretation, or in all these areas; took part in drafting, revising or critically reviewing the article; gave final approval of the version to be published; have agreed on the journal to which the article has been submitted; and agree to be accountable for all aspects of the work.

\section{Funding}

This work was supported by the National Natural Science Foundation of China [grant number BK20161386], Natural Science Foundation of Jiangsu [grant number BK20161386], Jiangsu Provincial Social Development Key Projects - Clinical Frontier Technologies [Grant Number BE2019719], Jiangsu Provincial Social Development - General Program [Grant Number BE20197180], Jiangsu Provincial Social Development General Program [Grant Number BE20197180], the Youth Program of Jiangsu Provincial Natural Scientific Foundation (No. BK20190129).

\section{Disclosure}

All authors report no conflicts of interest in this work.

\section{References}

1. Chen W, Zheng R, Baade PD, et al. Cancer statistics in China, 2015. CA Cancer J Clin. 2016;66(2):115-132. doi:10.3322/caac.21338

2. Cheng H, Perez-Soler R. Leptomeningeal metastases in non-smallcell lung cancer. Lancet Oncol. 2018;19(1):e43-e55. doi:10.1016/ S1470-2045(17)30689-7

3. Gleissner B, Chamberlain MC. Neoplastic meningitis. Lancet Neurol. 2006;5(5):443-452. doi:10.1016/S1474-4422(06)70443-4

4. Brower JV, Saha S, Rosenberg SA, et al. Management of leptomeningeal metastases: prognostic factors and associated outcomes. J Clin Neurosci. 2016;27:130-137. doi:10.1016/j.jocn.2015.11.012

5. Grossman SA, Krabak MJ. Leptomeningeal carcinomatosis. Cancer Treat Rev. 1999;25(2):103-119. doi:10.1053/ctrv.1999.0119

6. Luo W, Rao M, Qu J, Luo D. Applications of liquid biopsy in lung cancer-diagnosis, prognosis prediction, and disease monitoring. $\mathrm{Am}$ $J$ Transl Res. 2018;10(12):3911-3923.

7. Toh WS, Lai RC, Zhang B, Lim SK. MSC exosome works through a protein-based mechanism of action. Biochem Soc Trans. 2018;46 (4):843-853. doi:10.1042/BST20180079

8. Fan Y, Zhu X, Xu Y, et al. Cell-cycle and DNA-damage response pathway is involved in leptomeningeal metastasis of non-small cell lung cancer. Clin Cancer Res. 2018;24(1):209-216. doi:10.1158/ 1078-0432.CCR-17-1582

9. Zhang Q, Hu H, Liu H, et al. RNA sequencing enables systematic identification of platelet transcriptomic alterations in NSCLC patients. Biomed Pharmacother. 2018;105:204-214. doi:10.1016/j. biopha.2018.05.074

10. Coleman BM, Hanssen E, Lawson VA, Hill AF. Prion-infected cells regulate the release of exosomes with distinct ultrastructural features. FASEB J. 2012;26(10):4160-4173. doi:10.1096/fj.11-202077

11. Jiang B-Y, Yangsi L, Xue W, et al. Identification of leptomeningeal metastasis-specific exosomal miRNA signature in cerebrospinal fluid of non-small-cell lung cancer patients. J Clin Oncol. 2018;36 (15_suppl):e21024-e21024. doi:10.1200/JCO.2018.36.15_suppl. e21024

12. Rashed H, Bayraktar EK, Helal G, et al. Exosomes: from Garbage Bins to Promising Therapeutic Targets. Int J Mol Sci. 2017;18 (3):538. doi:10.3390/ijms18030538

13. Boukouris S, Mathivanan S. Exosomes in bodily fluids are a highly stable resource of disease biomarkers. Proteomics Clin Appl. 2015;9 (3-4):358-367. doi:10.1002/prca.201400114 
14. King HW, Michael MZ, Gleadle JM. Hypoxic enhancement of exosome release by breast cancer cells. BMC Cancer. 2012;12(1):421. doi:10.1186/1471-2407-12-421

15. Haderk F, Schulz R, Iskar M, et al. Tumor-derived exosomes modulate PD-L1 expression in monocytes. Sci Immunol. 2017;2(13) eaah5509. doi:10.1126/sciimmunol.aah5509

16. Becker A, Thakur BK, Weiss JM, Kim HS, Peinado H, Lyden D. Extracellular vesicles in cancer: cell-to-cell mediators of metastasis. Cancer Cell. 2016;30(6):836-848. doi:10.1016/j.ccell.2016.10.009

17. Hong CS, Funk S, Muller L, Boyiadzis M, Whiteside TL. Isolation of biologically active and morphologically intact exosomes from plasma of patients with cancer. $J$ Extracell Vesicles. 2016;5:29289. doi:10.3402/jev.v5.29289

18. Ludwig S, Floros T, Theodoraki MN, et al. Suppression of lymphocyte functions by plasma exosomes correlates with disease activity in patients with head and neck cancer. Clin Cancer Res. 2017;23 (16):4843-4854. doi:10.1158/1078-0432.CCR-16-2819

19. Ricklefs FL, Alayo Q, Krenzlin H, et al. Immune evasion mediated by PD-L1 on glioblastoma-derived extracellular vesicles. Sci $\mathrm{Adv}$. 2018;4(3):eaar2766. doi:10.1126/sciadv.aar2766

20. Theodoraki MN, Yerneni SS, Hoffmann TK, Gooding WE, Whiteside TL. Clinical Significance of PD-L1 ${ }^{+}$exosomes in plasma of head and neck cancer patients. Clin Cancer Res. 2018;24 (4):896-905. doi:10.1158/1078-0432.CCR-17-2664

21. Yang Y, Li CW, Chan LC, et al. Exosomal PD-L1 harbors active defense function to suppress $\mathrm{T}$ cell killing of breast cancer cells and promote tumor growth. Cell Res. 2018;28(8):862-864. doi:10.1038/ s41422-018-0060-4

22. Whiteside TL. The effect of tumor-derived exosomes on immune regulation and cancer immunotherapy. Future Oncol. 2017;13 (28):2583-2592. doi:10.2217/fon-2017-0343

23. Song Q, Xu Y, Yang C, et al. miR-483-5p promotes invasion and metastasis of lung adenocarcinoma by targeting RhoGDI1 and ALCAM. Cancer Res. 2014;74(11):3031-3042. doi:10.1158/00085472.CAN-13-2193

24. Lécuyer MA, Saint-Laurent O, Bourbonnière L, et al. Dual role of ALCAM in neuroinflammation and blood-brain barrier homeostasis. Proc Natl Acad Sci U S A. 2017;114(4):E524-E533. doi:10.1073/ pnas. 1614336114

25. Yang F, Liao X, Tian Y, Li G. Exosome separation using microfluidic systems: size-based, immunoaffinity-based and dynamic methodologies. Biotechnol J. 2017;12(4):1600699. doi:10.1002/ biot.201600699

26. Milane L, Singh A, Mattheolabakis G, Suresh M, Amiji MM. Exosome mediated communication within the tumor microenvironment. J Control Release. 2015;219:278-294. doi:10.1016/j.jconrel.2015.06.029
27. Whiteside TL. Tumor-derived exosomes and their role in cancer progression. Adv Clin Chem. 2016;74:103-141.

28. Li Y, Yin Z, Fan J, Zhang S, Yang W. The roles of exosomal miRNAs and lncRNAs in lung diseases. Signal Transduct Target Ther. 2019;4 (1):47. doi:10.1038/s41392-019-0080-7

29. Gao F, Zhang YF, Zhang ZP, et al. miR-342-5p regulates neural stem cell proliferation and differentiation downstream to notch signaling in mice. Stem Cell Reports. 2017;8(4):1032-1045. doi:10.1016/j. stemcr.2017.02.017

30. Abbott NJ, Rönnbäck L, Hansson E. Astrocyte-endothelial interactions at the blood-brain barrier. Nat Rev Neurosci. 2006;7(1):41-53. doi: $10.1038 / \mathrm{nrn} 1824$

31. Dehouck MP, Méresse S, Delorme P, Fruchart JC, Cecchelli R. An easier, reproducible, and mass-production method to study the blood-brain barrier in vitro. J Neurochem. 1990;54(5):1798-1801. doi:10.1111/j.1471-4159.1990.tb01236.x

32. Rubin LL, Hall DE, Porter S, et al. A cell culture model of the blood-brain barrier. $J$ Cell Biol. 1991;115(6):1725-1735. doi:10.1083/jcb.115.6.1725

33. Zhou L, Lv T, Zhang Q, et al. The biology, function and clinical implications of exosomes in lung cancer. Cancer Lett. 2017;407:84-92. doi:10.1016/j.canlet.2017.08.003

34. Rodríguez M, Silva J, López-Alfonso A, et al. Different exosome cargo from plasma/bronchoalveolar lavage in non-small-cell lung cancer. Genes Chromosomes Cancer. 2014;53(9):713-724. doi:10.1002/gcc.22181

35. Zhang Y, Zhang Y, Yin Y, Li S. Detection of circulating exosomal miR-17-5p serves as a novel non-invasive diagnostic marker for non-small cell lung cancer patients. Pathol Res Pract. 2019;215 (8):152466. doi:10.1016/j.prp.2019.152466

36. Jin X, Chen Y, Chen H, et al. Evaluation of tumor-derived exosomal miRNA as potential diagnostic biomarkers for early-stage non-small cell lung cancer using next-generation sequencing. Clin Cancer Res. 2017;23(17):5311-5319. doi:10.1158/1078-0432.CCR-17-0577

37. Yuan X, Wu H, Han N, et al. Notch signaling and EMT in non-small cell lung cancer: biological significance and therapeutic application. J Hematol Oncol. 2014;7(1):87. doi:10.1186/s13045-014-0087-z

38. Zhang X, Sai B, Wang F, et al. Hypoxic BMSC-derived exosomal miRNAs promote metastasis of lung cancer cells via STAT3-induced EMT. Mol Cancer. 2019;18(1):40. doi:10.1186/s12943-019-0959-5

39. Simard M, Nedergaard M. The neurobiology of glia in the context of water and ion homeostasis. Neuroscience. 2004;129(4):877-896. doi:10.1016/j.neuroscience.2004.09.053
OncoTargets and Therapy

\section{Publish your work in this journal}

OncoTargets and Therapy is an international, peer-reviewed, open access journal focusing on the pathological basis of all cancers, potential targets for therapy and treatment protocols employed to improve the management of cancer patients. The journal also focuses on the impact of management programs and new therapeutic

Submit your manuscript here: https://www.dovepress.com/oncotargets-and-therapy-journal agents and protocols on patient perspectives such as quality of life, adherence and satisfaction. The manuscript management system is completely online and includes a very quick and fair peer-review system, which is all easy to use. Visit http://www.dovepress.com/ testimonials.php to read real quotes from published authors. 